\title{
An Introduction to Evolutionary Multi-objective Optimization with Some Applications in Pattern Recognition
}

\author{
Carlos A. Coello-Coello \\ CINVESTAV (Evolutionary Computation Group) \\ Departamento de Computación \\ Av. IPN No. 2508, Col. San Pedro Zacatenco \\ México, D.F. 07360, Mexico \\ ccoello@cs.cinvestav.mx
}

\begin{abstract}
In this paper, we provide a general introduction to the socalled multi-objective evolutionary algorithms, which are metaheuristic search techniques inspired on natural evolution that are able to deal with highly complex optimization problems having two or more objectives. In the first part of the paper, we provide some basic concepts necessary to make the paper self-contained, as well as a short review of the most representative multi-objective evolutionary algorithms currently available in the specialized literature. After that, a short review of applications of these algorithms in pattern recognition is provided. The final part of the paper presents some possible future research paths in this area as well as our conclusions.
\end{abstract}

\section{Introduction}

Many real-world applications involve the simultaneous optimization of two or more (usually conflicting) objectives. These are known as multi-objective optimization problems (MOPs), and their solution requires finding not one, but a set of solutions that represent the best possible trade-offs among the objectives to be optimized. These trade-off solutions constitute (in decision variable space) the so-called Pareto optimal set and their corresponding values in objective function space constitutes the so-called Pareto front.

Although a wide variety of mathematical programming techniques are currently available to solve MOPs of different types [1, such approaches have a number of limitations, including the fact that they are normally very susceptible to the specific features of the problem to be solved (e.g., to the shape or continuity of the Pareto front of the MOP). Additionally, these approaches tend to generate a single solution per run, and departing from different starting points could lead to the same final solution. Metaheuristic 1 are an alternative for

\footnotetext{
${ }^{1}$ A metaheuristic is a high level strategy for exploring search spaces by using different methods 2. Metaheuristics have both a diversification (i.e., exploration of the search space) and an intensification (i.e., exploitation of the accumulated search experience) procedure.
}

E. Bayro-Corrochano and E. Hancock (Eds.): CIARP 2014, LNCS 8827, pp. 1-13 2014.

(C) Springer International Publishing Switzerland 2014 
solving complex optimization problems, such as nonlinear MOPs, which is the reason why their use has become so popular in recent years [3].

Evolutionary Algorithms (EAs) are, with no doubt, the most popular bioinspired metaheuristic in current use. EAs simulate the evolutionary process in a computer and use it to solve highly complex problems [4. EAs are particularly suitable for solving MOPs, because they operate on a population of solutions, which makes possible to generate several elements of the Pareto optimal set in a single run, instead of only one (as done by most mathematical programming techniques). Additionally, EAs require less domain-specific information (e.g., they don't require that the objective functions are differentiable) and are less susceptible to the shape and continuity of the Pareto front than mathematical programming techniques.

The first actual Multi-Objective Evolutionary Algorithm (MOEA) was proposed by David Schaffer in the mid-1980s [5. However, it was until 10 years later that the study and development of MOEAs started to attract some serious attention from researchers. Today, there is a considerably large volume of publications on different aspects of MOEAs and their applications, available in the literature 2

The remainder of this paper is organized as follows. Section 2 provides some basic multi-objective optimization concepts required to make this paper selfcontained. A very short introduction to evolutionary algorithms is presented in Section 3, while Section 4 briefly describes the main MOEAs in current use. After that, Section 5 provides a short review of the possible application of MOEAs in three popular pattern recognition tasks (classification, image segmentation and feature selection). Then, Section [6] describes some potential paths for future research in this area. Finally, the conclusions of the paper are provided in Section 7

\section{Basic Concepts}

In this paper, we focus on the solution of problems of the type 3 :

$$
\text { minimize } \boldsymbol{f}(\boldsymbol{x}):=\left[f_{1}(\boldsymbol{x}), f_{2}(\boldsymbol{x}), \ldots, f_{k}(\boldsymbol{x})\right]
$$

subject to:

$$
\begin{gathered}
g_{i}(\boldsymbol{x}) \leq 0 \quad i=1,2, \ldots, m \\
h_{i}(\boldsymbol{x})=0 \quad i=1,2, \ldots, p
\end{gathered}
$$

where $\boldsymbol{x}=\left[x_{1}, x_{2}, \ldots, x_{n}\right]^{T}$ is the vector of decision variables, $f_{i}: \mathbb{R}^{n} \rightarrow \mathbb{R}, i=$ $1, \ldots, k$ are the objective functions and $g_{i}, h_{j}: \mathbb{R}^{n} \rightarrow \mathbb{R}, i=1, \ldots, m, j=1, \ldots, p$ are the constraint functions of the problem.

\footnotetext{
${ }^{2}$ The author maintains the EMOO repository, which currently contains over 8800 bibliographic references related to evolutionary multi-objective optimization. The EMOO repository is located at: http://delta.cs.cinvestav.mx/ ccoello/EMOO/

${ }^{3}$ Without loss of generality, we will only assume minimization problems.
} 
To describe the concept of optimality in which we are interested, we will introduce next a few definitions.

Definition 1. Given two vectors $\boldsymbol{x}, \boldsymbol{y} \in \mathbb{R}^{k}$, we say that $\boldsymbol{x} \leq \boldsymbol{y}$ if $x_{i} \leq y_{i}$ for $i=1, \ldots, k$, and that $\boldsymbol{x}$ dominates $\boldsymbol{y}$ (denoted by $\boldsymbol{x} \prec \boldsymbol{y}$ ) if $\boldsymbol{x} \leq \boldsymbol{y}$ and $\boldsymbol{x} \neq \boldsymbol{y}$.

Definition 2. We say that a vector of decision variables $\boldsymbol{x} \in \mathcal{X} \subset \mathbb{R}^{n}$ is nondominated with respect to $\mathcal{X}$, if there does not exist another $\boldsymbol{x}^{\prime} \in \mathcal{X}$ such that $\boldsymbol{f}\left(\boldsymbol{x}^{\prime}\right) \prec \boldsymbol{f}(\boldsymbol{x})$.

Definition 3. We say that a vector of decision variables $\boldsymbol{x}^{*} \in \mathcal{F} \subset \mathbb{R}^{n}(\mathcal{F}$ is the feasible region) is Pareto-optimal if it is nondominated with respect to $\mathcal{F}$.

Definition 4. The Pareto Optimal Set $\mathcal{P}^{*}$ is defined by:

$$
\mathcal{P}^{*}=\{\boldsymbol{x} \in \mathcal{F} \mid \boldsymbol{x} \text { is Pareto-optimal }\}
$$

Definition 5. The Pareto Front $\mathcal{P F}^{*}$ is defined by:

$$
\mathcal{P} \mathcal{F}^{*}=\left\{\boldsymbol{f}(\boldsymbol{x}) \in \mathbb{R}^{k} \mid \boldsymbol{x} \in \mathcal{P}^{*}\right\}
$$

Therefore, our aim is to determine the Pareto optimal set from the set $\mathcal{F}$ of all the decision variable vectors that satisfy (2) and (3). It is worth noticing, however, that in practice, not all the Pareto optimal set may be desirable (or even achievable).

\section{A Very Short Introduction to Evolutionary Algorithms}

Although today it is more common to use the term "evolutionary algorithm" in a generic sense, in their origins, there were three main types of approaches: genetic algorithms [6, evolution strategies [7] and evolutionary programming [8. Each of them were developed by independent researchers who had different motivations, and aimed to solve a specific type of problem. However, over the years, the specific features of each of these three types of EAs were combined in different ways, eventually leading to a generic type of EA, whose main feature is that it uses a selection mechanism based on a fitness measure (which provides a measure of relative performance of solutions with respect to each other) that is applied on a set (called "population") of solutions (called "individuals").

As indicated before, the specific features of EAs have made them a good choice for solving MOPs, and their use has considerably increased in the last 20 years [3].

Multi-objective Evolutionary Algorithms (MOEAs) differ from traditional (single-objective) EAs in two main aspects:

- Selection Mechanism: EAs aim to maximize (or minimize) fitness values. In contrast, MOEAs aim to generate as many different nondominated solutions as possible. Pareto optimality has normally been adopted for this 
sake [4910, but other mechanisms are also possible (e.g., indicator-based selection [1]).

- Diversity Maintenance: Since MOEAs aim to produce as many different elements of the Pareto optimal set as possible, in a single run, they require a mechanism that avoids convergence to a single solution. The most popular diversity maintenance mechanisms include: fitness sharing and niching 1213, clustering [1415], geographically-based schemes [16], and the use of entropy 17,18.

An additional component of modern MOEAs is elitism, which refers to retaining the best solutions found at each generation, so that they are not destroyed by the genetic operators (e.g., crossover and mutation). In MOEAs, the most popular elitist schemes are external archives [16] (also called secondary populations) and the use of a "plus" selection scheme in which the population of parents is combined with the population of offspring, in such a way that the best half of this union constitutes the population at the following generation [19.

It is worth noticing that the use of an external archive is the most popular elitist mechanism, since such an archive allows to implement diversity maintenance mechanisms [20], as well as proving convergence, under certain assumptions [21.

\section{Multi-Objective Evolutionary Algorithms}

Although there is a wide variety of MOEAs available in the specialized literature, only a handful of them are in wide use. The following are, in the view of the author, the most representative MOEAs in current use:

1. Nondominated Sorting Genetic Algorithm II (NSGA-II): This is a revised and considerably improved version of one of the earliest MOEAs, called Nondominated Sorting Genetic Algorithm (NSGA), which was propsoed in the mid 1990s [22]. The NSGA-II uses a Pareto ranking procedure which is more efficient (computationally speaking) than the one adopted in the original NSGA. Also, it estimates the density of solutions surrounding a particular individual in the population by computing the average distance of two points on either side of this point along each of the objectives of the problem. This value is called crowding distance and is easy and computationally efficient to compute, without requiring any user-defined parameter. During the selection stage, NSGA-II uses a crowded-comparison operator that takes into consideration both the nondomination rank of an individual and its crowding distance, in such a way that nondominated solutions are preferred over dominated solutions, but between two solutions with the same nondomination rank, the one that resides in the less crowded region is preferred. Unlike most modern MOEAs, NSGA-II does not use an external archive to store the solutions found during the evolutionary process. Instead, it combines the best parents with the best offspring produced at each generation and keeps the best half of this union. Its clever mechanisms make the NSGA-II a very efficient algorithm which is also easy to use. Additionally, 
its source code is available in the public domain. These reasons have made it the most popular MOEA in the specialized literature for more than 10 years.

2. Strength Pareto Evolutionary Algorithm (SPEA): This MOEA was developed in the late 1990s, with the idea of merging the ideas of the several MOEAs available at that time. Its distinctive feature is the use of an external archive (called the external nondominated set), that stores the nondominated solutions generated during the search, and that is used (in combination with the main population of the evolutionary algorithm) during the selection process. SPEA computes a strength value for each individual, which is proportional to the number of solutions that dominate it. The size of the external nondominated set can have a significant growth during the evolutionary process, which dilutes the selection pressure. Because of this, the authors of SPEA decided to use a clustering technique to prune the contents of the external nondominated set so that its size does not exceed a certain (pre-defined) value. In 2001, a revised version of this algorithm called Strength Pareto Evolutionary Algorithm 2 (SPEA2) was introduced. SPEA2 has three main differences with respect to its predecessor [23]: (1) it incorporates a fine-grained fitness assignment strategy which takes into account, for each individual, the number of individuals that dominate it and the number of individuals by which it is dominated; (2) it uses a nearest neighbor density estimation technique which guides the search more efficiently, and (3) it has an enhanced archive truncation method that guarantees the preservation of boundary solutions.

\section{Multiobjective Evolutionary Algorithm Based on Decomposition} (MOEA/D): This approach was introduced in 2007 24, and it adopts a scalarization approach. The main idea of MOEA/D is to decompose a MOP into several scalar optimization sub-problems which are simultaneously optimized. During the optimization of each sub-problem, only information from the neighboring sub-problems is used, which allows this algorithm to be effective and efficient. In fact, MOEA/D can be seen as a successful example of the incorporation of mathematical programming techniques into a MOEA. The authors of MOEA/D have provided evidence of the good performance of this MOEA with respect to other approaches such as NSGA-II, and over the years, a number of variants of this algorithm have been introduced in the specialized literature (see for example [25 26 27] ).

Many other MOEAs are currently available (see for example [282930]), but none of them is widely used in the literature. This, however, has not discouraged algorithm developers who have now focused their efforts on aspects such as computational efficiency [31] and scalability [11/32].

One of the most intriguing and interesting topics currently under research in algorithmic design is the use of selection mechanisms based on performance indicators. IBEA 28] was the first algorithm of this sort, but several others have been proposed over the years (see for example [113334]). 


\section{Some Applications in Pattern Recognition}

MOEAs have been widely used in pattern recognition (see for example [35|36]) and this paper does not intend, by any means, to provide a survey on this sort of research. For illustrative purposes only, we will describe next three types of common applications on MOEAs in pattern recognition:

1. Classification: In this task, each input value is placed into a class (from several available), based on information provided by its descriptors. When treated as a MOP, classification normally involves objectives such as minimizing the complexity of the classifier (e.g., the number of rules that it contains) while maximizing its accuracy (i.e., minimizing the classification error). However, it is possible to adopt other objectives such as the generality of the rules, their understandability or their complexity.

For example, Zheng et al. 37] maximized both precision (a measure of exactness in the classification performed) and recall (a measure of completeness) in a problem in which the goal was to mine rules for classifying an evacuee population during a fire using a multi-objective particle swarm optimizer. The aim of this application was to improve evacuation decisions and save lives.

In contrast, Suttorp and Igel 38 minimized three objectives: (1) the false positive rate, (2) the false negative rate and (3) the number of support vectors in an application related to online pedestrian detection in infrared images for driver assistance systems. The authors indicated that in this application, the Pareto front of the first two objectives could be viewed as a ROC curve in which each point corresponded to a learning machine optimized for a particular trade-off between sensitivity and specificity. The third objective (number of support vectors) was aimed to reduce the model complexity, which was a concern in this application, due to the existence of real-time constraints.

One of the main advantages of using MOEAs in classification is that they can overcome problems commonly associated to traditional (i.e., single-objective) classifiers, such as overfitting/overlearning and disregarding small classes.

2. Image Segmentation: The term "segmentation" refers to the clustering of the pixels of an image based on certain criteria. The output of a segmentation process is usually another image with raw pixel data, which constitutes either the boundary of a region or all the points in the region itself. Image segmentation is, in general, a very challenging task. When posed as a MOP, image segmentation can involve several different objectives [39].

For example, in Mukhopadhyay and Maulik [40, two objectives were considered: (1) minimize fuzzy compactness of the clusters and (2) maximize the fuzzy separation among the clusters. In this application, a multi-objective (real-coded) genetic fuzzy clustering scheme was adopted for the segmentation of multispectral magnetic resonance images (MRI) of the human brain. The search engine adopted in this case was an approach proposed by the 
authors, which was called multiobjective variable string length genetic fuzzy clustering algorithm (MOVGA), which used NSGA-II [19] as its underlying multi-objective framework.

In contrast, Bhanu and Lee 41 considered five objectives when applying a genetic algorithm with a linear aggregating function to an image segmentation problem: (1) edge-border coincidence, (2) boundary consistency, (3) pixel classification, (4) object overlap, and (5) object contrast.

Nevertheless, and regardless of the objective functions adopted, MOEAs offer the advantage of allowing the generation of several output images, representing different trade-offs among the objectives. This provides the decision maker with more alternatives to choose from, as opposed to single-objective techniques, that produce as an output, a single image.

3. Feature Selection: It refers to the extraction of features for differentiating one class of objects from another. The output of this process is a vector of values of the measured features. Feature selection can be done with a supervised or with an unsupervised approach. In the supervised case, the existence of a training set of objectives for which the actual class labels are known, is assumed. In contrast, in the unsupervised case, the existence of true class labels is not assumed and, therefore, there is no training set. Most approaches that treat feature selection as a MOP, assume the supervised case [35. For example, in Mendes et al. 42, two objectives are considered: (1) minimize the number of features and (2) maximize the accuracy. The authors used in this case two classifiers: support vector machines and a logistic function. Both were applied to a database containing financial statements of 1200 medium-size private French companies, in an application related to bankruptcy prediction.

Unsupervised cases are relatively rare in multiobjective feature selection applications. For example, Morita et al. 43 used the NSGA [22] in unsupervised learning applied to handwritten word recognition tasks. In this case, two objectives were minimized: (1) the number of features and (2) a validity index that measured the quality of clusters. A standard $k$-means algorithm was then applied to form the given number of clusters based on the selected features and the number of selected clusters.

The above examples illustrate the flexibility that the use of MOEAs can introduce, in general, when applied to pattern recognition tasks.

\section{Potential Areas for Further Research}

From the author's perspective, there are several research paths that are worth exploring regarding the application of MOEAs in pattern recognition. For example:

- Integration: Clearly, the use of MOEAs to develop fully-automated pattern recognition systems seems a realistic (perhaps long-term) goal. Such systems 
could be applied to different types of databases with little (or no) human intervention. It is likely that the development of such systems may require the hybridization of MOEAs with other techniques such as fuzzy logic and/or machine learning approaches. Additionally, this task may require the design of new architectures that allow an efficient and effective integration of different types of approaches during the different stages involved in a pattern recognition task (see for example [4]). Evidently, MOEAs are a very suitable choice for this sort of task, because of their ability to deal with several conflicting objectives at the same time.

- Efficiency: One of the main criticisms that normally arises when using MOEAs in real-world problems is that they usually require of a high computational cost for producing reasonably good results, when the objective functions are computationally expensive. This is of particular relevance in certain pattern recognition tasks, such as image segmentation. However, it is possible to reduce the computational cost of MOEAs using techniques such as: fitness approximation [45], fitness inheritance [46], parallelization 4748] and surrogate methods 49 . Nevertheless, the use of such techniques in this area is still relatively scarce.

- Use of other Metaheuristics: A variety of other bio-inspired metaheuristics have become popular in the last few years [50], and, in fact, many of them have already been applied to pattern recognition tasks. The following is a non-comprehensive list of bio-inspired metaheuristics that, from the author's perspective, could be useful in pattern recognition tasks:

- Artificial Immune Systems (AIS): Our natural immune system has provided a fascinating metaphor for developing a new bio-inspired metaheuristic. Indeed, from a computational point of view, our immune system can be considered as a highly parallel intelligent system that is able to learn and retrieve previously acquired knowledge (i.e., it has "memory"), when solving highly complex recognition and classification tasks. This motivated the development of the so-called artificial immune systems (AISs) during the early 1990s [5152]. AISs have been already used for classification and pattern recognition tasks, in general (see for example [53]), mainly because of their suitability for these problems (recognizing an antigen that invades our blood system is, after all, a pattern recognition task that our immune system solves on an daily basis). Nevertheless, the use of multi-objective AISs in pattern recognition tasks is still relatively rare (see for example [54]).

- Particle Swarm Optimization (PSO): This metaheuristic is inspired on the movements of a flock of birds seeking food, and it was proposed in the mid-1990s [55. In the PSO algorithm, the behavior of each particle (i.e., individual) is affected by either the best local (within a certain neighborhood) or the best global (i.e., with respect to the entire swarm, 
or population) individual. PSO allows particles to benefit from their past experiences (a mechanism that doesn't exist in traditional evolutionary algorithms) and uses neighborhood structures that can regulate the behavior of the algorithm. The use of multi-objective particle swarm optimizers in pattern recognition tasks has been more frequent than that of other bio-inspired metaheuristics (see for example [56.37]), but the full potential of multi-objective PSO approaches in this area is still to be exploited.

- Ant Colony Optimization (ACO): This metaheuristic was inspired on the behavior observed in colonies of real ants seeking for food. Ants deposit a chemical substance on the ground, called pheromone [57, which influences the behavior of the ants: they tend to take those paths in which there is a larger amount of pheromone. Therefore, pheromone trails can be seen as an indirect communication mechanism used by the ants (which can be seen as agents that interact to solve complex tasks). This interesting behavior of ants gave rise to a metaheuristic called ant system, which was originally applied to the travelling salesperson problem. Nowadays, the several variations of this algorithm that have been developed over the years, are collectively denominated ant colony optimization (ACO), and they have been applied to a wide variety of problems, including some pattern recognition tasks. However, its use in multi-objective pattern recognition tasks is still rare (see for example [58]).

\section{Conclusions}

This paper has attempted to provide a general overview of multi-objective evolutionary algorithms and some of their possible applications in pattern recognition. The paper included a short introduction to evolutionary computation, as well as some basic definitions related to multi-objective optimization. Also, some of the main MOEAs in current use have been briefly described. In the last part of the paper, some possible paths for future research in this area were briefly discussed.

The main goal of this paper is to motivate experts in pattern recognition to adopt MOEAs as an additional tool for their research work, mainly because of their flexibility and the several advantages that they can offer to this field.

Acknowledgements. The author acknowledges support from CONACyT through project 221551 .

\section{References}

1. Miettinen, K.M.: Nonlinear Multiobjective Optimization. Kluwer Academic Publishers, Boston, Massachusetts (1999)

2. Blum, C., Roli, A.: Metaheuristics in combinatorial optimization: Overview and conceptual comparison. ACM Computing Surveys 35(3), 268-308 (2003) 
3. Coello Coello, C.A., Lamont, G.B., Van Veldhuizen, D.A.: Evolutionary Algorithms for Solving Multi-Objective Problems, 2nd edn. Springer, New York (2007) ISBN 978-0-387-33254-3

4. Goldberg, D.E.: Genetic Algorithms in Search, Optimization and Machine Learning. Addison-Wesley Publishing Company, Reading (1989)

5. Schaffer, J.D.: Multiple Objective Optimization with Vector Evaluated Genetic Algorithms. In: Genetic Algorithms and their Applications: Proceedings of the First International Conference on Genetic Algorithms, Lawrence Erlbaum, pp. 93$100(1985)$

6. Holland, J.H.: Concerning efficient adaptive systems. In: Yovits, M.C., Jacobi, G.T., Goldstein, G.D. (eds.) Self-Organizing Systems-1962, pp. 215-230. Spartan Books, Washington, D.C (1962)

7. Schwefel, H.P.: Kybernetische evolution als strategie der experimentellen forschung in der strömungstechnik. Dipl.-Ing. thesis (1965) (in German)

8. Fogel, L.J.: Artificial Intelligence through Simulated Evolution. John Wiley, New York (1966)

9. Fonseca, C.M., Fleming, P.J.: Genetic Algorithms for Multiobjective Optimization: Formulation, Discussion and Generalization. In: Forrest, S. (ed.) Proceedings of the Fifth International Conference on Genetic Algorithms, San Mateo, California, University of Illinois at Urbana-Champaign, pp. 416-423. Morgan Kauffman Publishers (1993)

10. Srinivas, N., Deb, K.: Multiobjective optimization using nondominated sorting in genetic algorithms. Technical report, Department of Mechanical Engineering, Indian Institute of Technology, Kanpur, India (1993)

11. Beume, N., Naujoks, B., Emmerich, M.: SMS-EMOA: Multiobjective selection based on dominated hypervolume. European Journal of Operational Research 181(3), 1653-1669 (2007)

12. Goldberg, D.E., Richardson, J.: Genetic algorithm with sharing for multimodal function optimization. In: Grefenstette, J.J. (ed.) Genetic Algorithms and Their Applications: Proceedings of the Second International Conference on Genetic Algorithms, Hillsdale, New Jersey, pp. 41-49. Lawrence Erlbaum (1987)

13. Deb, K., Goldberg, D.E.: An Investigation of Niche and Species Formation in Genetic Function Optimization. In: Schaffer, J.D. (ed.) Proceedings of the Third International Conference on Genetic Algorithms, San Mateo, California, George Mason University, pp. 42-50. Morgan Kaufmann Publishers (June 1989)

14. Toscano Pulido, G., Coello Coello, C.A.: Using Clustering Techniques to Improve the Performance of a Multi-objective Particle Swarm Optimizer. In: Deb, K., Tari, Z. (eds.) GECCO 2004. LNCS, vol. 3102, pp. 225-237. Springer, Heidelberg (2004)

15. Zitzler, E., Deb, K., Thiele, L.: Comparison of Multiobjective Evolutionary Algorithms on Test Functions of Different Difficulty. In: Wu, A.S. (ed.) Proceedings of the 1999 Genetic and Evolutionary Computation Conference on Workshop Program, Orlando, Florida, pp. 121-122 (July 1999)

16. Knowles, J., Corne, D.: Properties of an Adaptive Archiving Algorithm for Storing Nondominated Vectors. IEEE Transactions on Evolutionary Computation 7(2), 100-116 (2003)

17. Kita, H., Yabumoto, Y., Mori, N., Nishikawa, Y.: Multi-Objective Optimization by Means of the Thermodynamical Genetic Algorithm. In: Ebeling, W., Rechenberg, I., Voigt, H.-M., Schwefel, H.-P. (eds.) PPSN 1996. LNCS, vol. 1141, pp. 504-512. Springer, Heidelberg (1996) 
18. Cui, X., Li, M., Fang, T.: Study of Population Diversity of Multiobjective Evolutionary Algorithm Based on Immune and Entropy Principles. In: Proceedings of the Congress on Evolutionary Computation 2001 (CEC 2001), vol. 2, pp. 1316-1321. IEEE Service Center, Piscataway (2001)

19. Deb, K., Pratap, A., Agarwal, S., Meyarivan, T.: A Fast and Elitist Multiobjective Genetic Algorithm: NSGA-II. IEEE Transactions on Evolutionary Computation 6(2), 182-197 (2002)

20. Knowles, J.D., Corne, D.W.: Approximating the Nondominated Front Using the Pareto Archived Evolution Strategy. Evolutionary Computation 8(2), 149-172 (2000)

21. Schuetze, O., Laumanns, M., Tantar, E., Coello Coello, C.A., Talbi, E.G.: Computing Gap Free Pareto Front Approximations with Stochastic Search Algorithms. Evolutionary Computation 18(1), 65-96 (2010)

22. Srinivas, N., Deb, K.: Multiobjective Optimization Using Nondominated Sorting in Genetic Algorithms. Evolutionary Computation 2(3), 221-248 (1994)

23. Zitzler, E., Laumanns, M., Thiele, L.: SPEA2: Improving the Strength Pareto Evolutionary Algorithm. In: Giannakoglou, K., Tsahalis, D., Periaux, J., Papailou, P., Fogarty, T. (eds.) Evolutionary Methods for Design, Optimization and Control with Applications to Industrial Problems, EUROGEN 2001, Athens, Greece, pp. 95-100 (2002)

24. Zhang, Q., Li, H.: MOEA/D: A Multiobjective Evolutionary Algorithm Based on Decomposition. IEEE Transactions on Evolutionary Computation 11(6), 712-731 (2007)

25. Chen, C.M., Ping Chen, Y., Zhang, Q.: Enhancing MOEA/D with Guided Mutation and Priority Update for Multi-Objective Optimization. In: 2009 IEEE Congress on Evolutionary Computation (CEC 2009), Trondheim, Norway, pp. 209216. IEEE Press (May 2009)

26. Chiang, T.C., Lai, Y.P.: MOEA/D-AMS: Improving MOEA/D by an Adaptive Mating Selection Mechanism. In: 2011 IEEE Congress on Evolutionary Computation (CEC 2011), New Orleans, Lousiana, USA, June 5-8, pp. 1473-1480. IEEE Service Center (2011)

27. Qi, Y., Ma, X., Liu, F., Jiao, L., Sun, J., Wu, J.: MOEA/D with Adaptive Weight Adjustment. Evolutionary Computation 22(2), 231-264 (2014)

28. Zitzler, E., Künzli, S.: Indicator-based Selection in Multiobjective Search. In: Yao, X., et al. (eds.) PPSN 2004. LNCS, vol. 3242, pp. 832-842. Springer, Heidelberg (2004)

29. Deb, K., Mohan, M., Mishra, S.: Evaluating the $\epsilon$-Domination Based MultiObjective Evolutionary Algorithm for a Quick Computation of Pareto-Optimal Solutions. Evolutionary Computation 13(4), 501-525 (2005)

30. Toscano Pulido, G., Coello Coello, C.A.: The Micro Genetic Algorithm 2: Towards Online Adaptation in Evolutionary Multiobjective Optimization. In: Fonseca, C.M., Fleming, P.J., Zitzler, E., Deb, K., Thiele, L. (eds.) EMO 2003. LNCS, vol. 2632, pp. 252-266. Springer, Heidelberg (2003)

31. Knowles, J.: ParEGO: A Hybrid Algorithm With On-Line Landscape Approximation for Expensive Multiobjective Optimization Problems. IEEE Transactions on Evolutionary Computation 10(1), 50-66 (2006)

32. Bader, J., Zitzler, E.: HypE: An Algorithm for Fast Hypervolume-Based ManyObjective Optimization. Evolutionary Computation 19(1), 45-76 (2011) 
33. Rodríguez Villalobos, C.A., Coello Coello, C.A.: A New Multi-Objective Evolutionary Algorithm Based on a Performance Assessment Indicator. In: 2012 Genetic and Evolutionary Computation Conference (GECCO 2012), Philadelphia, USA, pp. 505-512. ACM Press (July 2012) ISBN: 978-1-4503-1177-9

34. Hernández Gómez, R., Coello Coello, C.A.: MOMBI: A New Metaheuristic for Many-Objective Optimization Based on the $R 2$ Indicator. In: 2013 IEEE Congress on Evolutionary Computation (CEC 2013), Cancún, México, June 20-23, pp. 24882495. IEEE Press (2013) ISBN 978-1-4799-0454-9

35. Mukhopadhyay, A., Maulik, U., Bandyopadhyay, S., Coello, C.A.C.: A Survey of Multiobjective Evolutionary Algorithms for Data Mining: Part I. IEEE Transactions on Evolutionary Computation 18(1), 4-19 (2014)

36. Mukhopadhyay, A., Maulik, U., Bandyopadhyay, S., Coello, C.A.C.: A Survey of Multiobjective Evolutionary Algorithms for Data Mining: Part II. IEEE Transactions on Evolutionary Computation 18(1), 20-35 (2014)

37. Zheng, Y.J., Ling, H.F., Xue, J.Y., Chen, S.Y.: Population Classification in Fire Evacuation: A Multiobjective Particle Swarm Optimization Approach. IEEE Transactions on Evolutionary Computation 18(1), 70-81 (2014)

38. Suttorp, T., Igel, C.: Multi-Objective Optimization of Support Vector Machines. In: Jin, Y. (ed.) Multi-Objective Machine Learning. SCI, vol. 16, pp. 199-220. Springer, Heidelberg (2006)

39. Chin-Wei, B., Rajeswari, M.: Multiobjective Optimization Approaches in Image Segmentation-The Directions and Challenges. International on Advances in Soft Computing and its Applications 2(1), 40-65 (2010)

40. Mukhopadhyay, A., Maulik, U.: A multiobjective approach to MR brain image segmentation. Applied Soft Computing 11(1), 872-880 (2011)

41. Bhanu, B., Lee, S.: Genetic Learning for Adaptive Image Segmentation. Kluwer Academic Publishers, Boston (1994)

42. Mendes, F., Duarte, J., Vieira, A., Gaspar-Cunha, A.: Feature Selection for Bankruptcy Prediction: A Multi-Objective Optimization Approach. In: Gao, X.Z., Gaspar-Cunha, A., Köppen, M., Schaefer, G., Wang, J. (eds.) Soft Computing in Industrial Applications. AISC, vol. 75, pp. 109-115. Springer, Heidelberg (2010)

43. Morita, M., Sabourin, R., Bortolozzi, F., Suen, C.: Unsupervised Feature Selection Using Multi-Objective Genetic Algorithm for Handwritten Word Recognition. In: Proceedings of the 7th International Conference on Document Analysis and Recognition (ICDAR 2003), Edinburgh, Scotland, pp. 666-670 (August 2003)

44. Guo, P.F., Bhattacharya, P., Kharma, N.: An Efficient Image Pattern Recognition System Using an Evolutionary Search Strategy. In: Proceedings of the 2009 IEEE International Conference on Systems, Man, and Cybernetics, San Antonio, Texas, USA. IEEE Press (October 2009)

45. Jin, Y.: A comprehensive survey of fitness approximation in evolutionary computation. Soft Computing 9(1), 3-12 (2005)

46. Reyes Sierra, M., Coello Coello, C.A.: Fitness Inheritance in Multi-Objective Particle Swarm Optimization. In: 2005 IEEE Swarm Intelligence Symposium (SIS 2005), Pasadena, California, USA, pp. 116-123. IEEE Press (June 2005)

47. López Jaimes, A., Coello Coello, C.A.: MRMOGA: A New Parallel Multi-Objective Evolutionary Algorithm Based on the Use of Multiple Resolutions. Concurrency and Computation: Practice and Experience 19(4), 397-441 (2007)

48. Sharma, D., Collet, P.: GPGPU-Compatible Archive Based Stochastic Ranking Evolutionary Algorithm (G-ASREA) for Multi-Objective Optimization. In: Schaefer, R., Cotta, C., Kołodziej, J., Rudolph, G. (eds.) PPSN XI. LNCS, vol. 6239, pp. 111-120. Springer, Heidelberg (2010) 
49. Lim, D., Jin, Y., Ong, Y.S., Sendhoff, B.: Generalizing Surrogate-Assisted Evolutionary Computation. IEEE Transactions on Evolutionary Computation 14(3), 329-355 (2010)

50. Corne, D., Dorigo, M., Glover, F. (eds.): New Ideas in Optimization. McGraw-Hill, London (1999)

51. Dasgupta, D. (ed.): Artificial Immune Systems and Their Applications. Springer, Berlin (1999)

52. de Castro, L.N., Timmis, J.: An Introduction to Artificial Immune Systems: A New Computational Intelligence Paradigm. Springer, London (2002) ISBN 1-85233-5947

53. Wang, W., Gao, S., Tang, Z.: Improved pattern recognition with complex artificial immune system. Soft Computing 13(12), 1209-1217 (2009)

54. Yang, D., Jiao, L., Gong, M., Liu, F.: Artificial immune multi-objective SAR image segmentation with fused complementary features. Information Sciences 181(13), 2797-2812 (2011)

55. Kennedy, J., Eberhart, R.C.: Swarm Intelligence. Morgan Kaufmann Publishers, San Francisco (2001)

56. Dehuri, S., Cho, S.B.: Multi-criterion Pareto based particle swarm optimized polynomial neural network for classification: A review and state-of-the-art. Computer Science Review 3(1), 19-40 (2009)

57. Dorigo, M., Stützle, T.: Ant Colony Optimization. The MIT Press (2004) ISBN 0-262-04219-3

58. He, Y., Chen, D., Zhao, W.: Integrated method of compromise-based ant colony algorithm and rough set theory and its application in toxicity mechanism classification. Chemometrics And Intelligent Laboratory Systems 92(1), 22-32 (2008) 